\title{
Ka-band Link Study and Analysis for a Mars Hybrid RF/Optical Software Defined Radio
}

\author{
Daniel J. Zeleznikar ${ }^{1}$, Jennifer M. Nappier ${ }^{2}$, and Joseph A. Downey ${ }^{3}$ \\ National Aeronautics and Space Administration \\ John H. Glenn Research Center at Lewis Field, Cleveland, Ohio 44135
}

\begin{abstract}
The integrated radio and optical communications (iROC) project at the NASA Glenn Research Center (GRC) is investigating the feasibility of a hybrid RF and optical communication subsystem for future deep space missions. The hybrid communications subsystem enables the advancement of optical communications while simultaneously mitigating the risk of infusion by combining an experimental optical transmitter and telescope with a reliable Ka-band RF transmitter and antenna. The iROC communications subsystem seeks to maximize the total data return over the course of a potential 2-year mission in Mars orbit beginning in 2021. Although optical communication by itself offers potential for greater data return over RF, the reliable Ka-band link is also being designed for high data return capability in this hybrid system. A daily analysis of the RF link budget over the 2-year span is performed to optimize and provide detailed estimates of the RF data return. In particular, the bandwidth dependence of these data return estimates is analyzed for candidate waveforms. In this effort, a data return modeling tool was created to analyze candidate RF modulation and coding schemes with respect to their spectral efficiency, amplifier output power back-off, required digital to analog conversion (DAC) sampling rates, and support by ground receivers. A set of RF waveforms is recommended for use on the iROC platform.
\end{abstract}

\section{Nomenclature}

$E_{b} / N_{0} \quad=$ energy-per-bit-to-noise-power-spectral-density ratio, $\mathrm{dB}-\mathrm{Hz}$

$\theta \quad=$ Earth elevation angle, degrees

\section{Introduction}

$\mathrm{O}$ ptical communications has offered potential for significantly higher data returns from NASA deep space missions for many years; however the risk associated with the unproven technology in the deep space environment has been a significant barrier to infusion into flight platforms. Since mission managers can reduce risk by relying on a legacy radio link in the communications subsystem, the integrated radio and optical communications (iROC) project is investigating a hybrid communications subsystem to allow demonstration of an optical capability while leveraging the confidence of a coupled RF system. A hybrid subsystem, as opposed to two separate subsystems, offers the potential to minimize the mass and power burden.

For the waveform portion of the hybrid communications subsystem, opportunities for integration exist at both the hardware and software level. In this context, waveform is defined as the set of transformations applied to information that is transmitted over the air and the corresponding set of transformations to convert received signals back to their information contents. ${ }^{1}$ For example, the RF and optical waveforms can be implemented on two separate hardware slices of the same "software-defined radio" (SDR), but the mass and power consumption might be better optimized if both waveforms could be processed in the same slice, or even using a shared processor. Utilizing the

\footnotetext{
${ }^{1}$ Electronics Engineer, Information and Signal Processing Branch, 21000 Brookpark Road, MS 54-4, AIAA Member.

${ }^{2}$ Computer Engineer, Information and Signal Processing Branch, 21000 Brookpark Road, MS 54-1.

${ }^{3}$ Computer Engineer, Information and Signal Processing Branch, 21000 Brookpark Road, MS 54-1.
} 
same modulation and coding formats for both the RF and optical links was also considered, however, a common this approach was deemed unsuitable due the distinctly different communications channels for deep-space optical and Ka-Band communications. Therefore, the modulation and coding choices for each link are optimized separately. This paper analyzes only the Ka-band link options.

A reliable RF capability in Ka-band (nominally $32 \mathrm{GHz}$ ) is proposed as a high performance data return link to NASA's deep space network (DSN). In this effort, the modulation and coding for an RF waveform that delivers the system's maximum data return on a daily basis is desired. Various tradeoffs between possible modulation formats and coding schemes have been analyzed significantly in prior work. In this study, the traditional tradeoffs are explored again, along with specific constraints related to the proposed Mars mission. A tool was created to perform day-by-day analysis of the links and to compare waveforms so that they could be optimized for data return within power and bandwidth constraints. Similar to previous studies, total mission data return is the driving metric used to recommend an RF waveform for use in a combined RF/optical software defined radio. This metric consists of the total amount of usable science data to be returned over the course of the 2-year mission timeframe.

Section II provides background information on iROC and its design reference mission, a review of a previous coding and modulation analysis performed by NASA in 2008, and an overview of prior studies conducted for KaBand return links. In Section III, the approach taken for iROC's Ka-band link and considerations included in the analysis tool are described. Results are presented and analyzed to compare the performance of different waveform options. Section IV provides a waveform recommendation for the iROC platform, including data return estimates for the proposed platform, and Section V presents the primary conclusions of the study.

\section{Background Information}

The iROC project chose the Mars Reconnaissance Orbiter (MRO) mission to use as a design reference mission on which to baseline the iROC telecommunications subsystem design. ${ }^{2}$ MRO was launched on August 12, 2005. The MRO primary mission operated over a two year time period, though the orbiter is still operating as of the date of this paper. The MRO mission included a primary X-Band telecommunications subsystem with an experimental Ka-Band capability. MRO's data return modem was the Small Deep Space Transponder (SDST), which has a peak data rate capability of $6 \mathrm{Mbps}$. MRO was expected to return 34 terabits (Tb) of science data over the course of the two-year mission, based on an X-band high gain antenna (HGA) with a gain of $46.7 \mathrm{dBi}$, pointing loss less than 0.3 $\mathrm{dB}$, and between 10 and 11 hours of total contact time per day to two of the DSN's 34-meter ground station antennas. ${ }^{3}$ In operation, the orbiter has achieved an average science data return of about $26 \mathrm{~Tb}$ per year ${ }^{4}$ since it entered into its science phase in late 2006 through late 2013, exceeding its initial success criteria by a factor of 2. Unfortunately, experimental use of the Ka-band link for data return during the mission's primary science phase was indefinitely postponed due to the failure of the primary SDST's Ka-band path. ${ }^{5}$

The expected mass of the iROC subsystem is being designed to not exceed that of the MRO telecommunications subsystem, and the power consumption of the iROC subsystem has a similar goal. The proposed iROC subsystem consists of a primary single fault tolerant Ka-Band RF communications capability for the uplink commands and the downlink telemetry and data with an experimental zero fault tolerant optical capability for additional high capacity downlink data only. A basic block diagram of the iROC downlink subsystem can be seen in Figure 1. The optical and RF waveforms will be implemented in an SDR. The iROC design includes a 75-watt traveling wave tube amplifier (TWTA) for Ka-Band and a 13-watt laser for the optical transmitter. The hybrid telescope/antenna used in iROC combines a co-bore-sighted 12.5-cm optical telescope within a 3-m antenna aperture, the same diameter as MRO's HGA. ${ }^{2}$

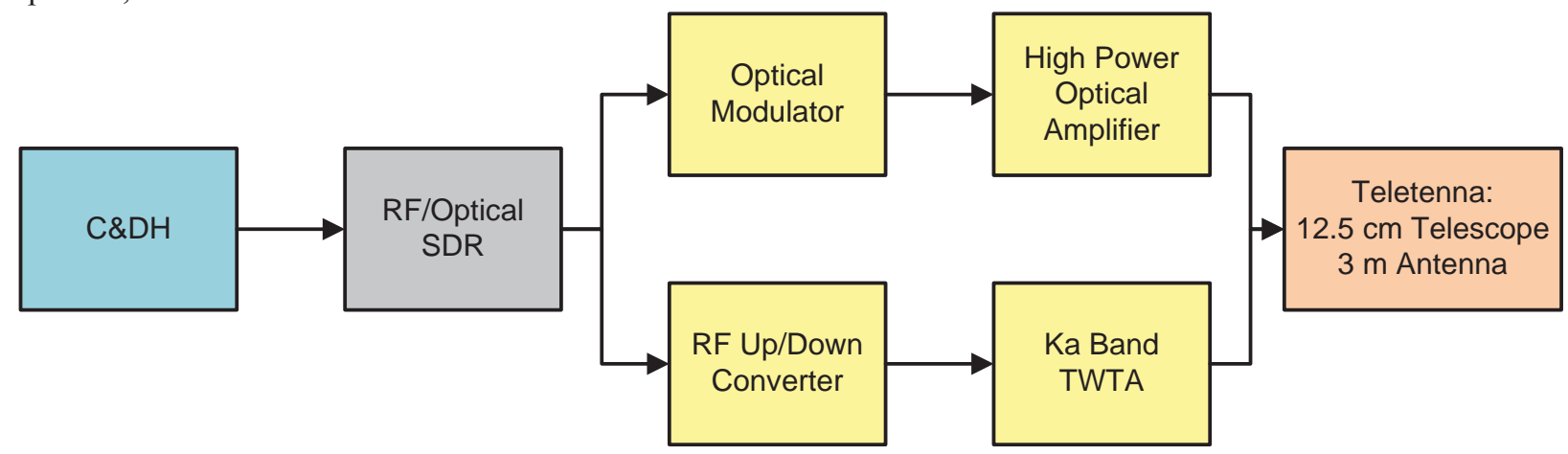

Figure 1. iROC subsystem functional block diagram. 
Similar to the MRO's initial two-year mission profile, Figure 2 shows the highly dynamic, but predictable nature of the communications link distance from Mars to Earth over the course of the proposed iROC mission: a two-year span, the same length as MRO's original mission. Over the two year epoch chosen for analysis, the link distance ranges from about 2.6 AU at its farthest point away from Earth, to about $0.5 \mathrm{AU}$ at its nearest point about 5 times closer. Large variation in the physical distance to Earth exists for all nearby planets in the inner solar system over the course of their orbital periods, which causes major variation in the free space path loss of the communications link which varies as the square of the distance. Mars exhibits some of the greatest path loss variation since its orbital radius is close to that of Earth.

The proposed iROC mission model predicts windows (or passes) during which RF and optical links can communicate with specific ground stations on the Earth without obstruction by Mars. Figure 2 shows the total daily visibilities over the course of the mission timeframe, reflecting the sum of the contact times from all possible windows in a given day to the DSN terminals at the Goldstone deep space communications complex, and likewise to the optical communication telescope laboratory (OCTL) telescope located at the Table Mountain Observatory about 100 miles away. For the RF links, contact times are considered only when the elevation angle of the ground station antenna is 10 degrees or higher. For the optical links, contact times are considered only when the elevation angle of the ground telescope is 30 degrees or higher. Notice how the contact times rise to maximum for both links asynchronous to the days during which the Earth and Mars are at their closest. This is because contact times are a function of Earth's inclination relative to the Earth-Mars reference vector as opposed to how far apart the two planets are. A period of solar conjunction where neither link can operate is also apparent near the beginning of the mission.

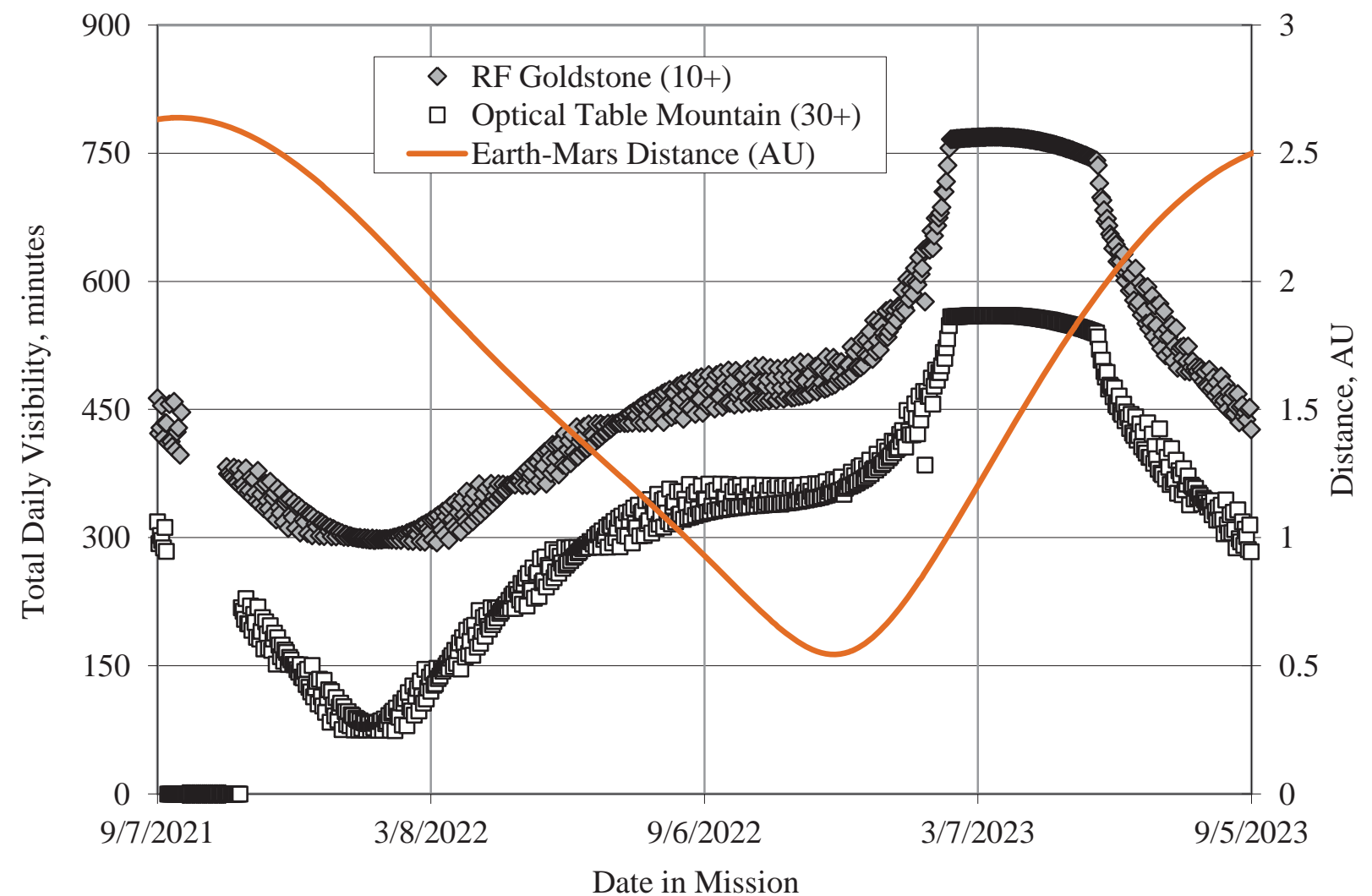

Figure 2. iROC total daily visibility from Mars to the Goldstone DSN and Table Mountain OCTL, including distance from MRO spacecraft to Earth over two-year mission.

The Coding and Modulation Link Protocol (CMLP) Study ${ }^{6}$ was conducted by NASA in 2008 and a set of modulation and coding schemes for deep space missions were recommended. The CMLP recommended pre-coded Gaussian Minimum Shift Keying (GMSK) ( $\mathrm{h}=0.5$ ), with a bandwidth-time (BT) product of 0.5 . This recommendation is reflected in current Space Frequency Coordination Group (SFCG) recommendations ${ }^{7}$, and a 
Consultative Committee on Space and Data Systems (CCSDS) recommended standard ${ }^{8}$. The CMLP recommended coding included LDPC and Turbo codes which are included in the CCSDS 131.4-M-1 Magenta Book ${ }^{9}$.

Several previous Ka-band link studies of interest also take the approach of data return maximization. A link study for the MRO Ka-band demonstration was released in $2005^{10}$. In a 2007 work by the same author ${ }^{11}$, a more complex approach for maximization of data return is presented. In a 2012 conference paper by a different author ${ }^{12}$, the availability optimization approach from Ref. 11 was applied to the Ka-band link for European Space Agency's BepiColombo mission. This paper's analysis follows a similar approach to maximizing data return with regards to changing data rates for varying link conditions.

\section{RF Link Study and Waveform Analysis}

A link budget tool was created to determine how various modulation and coding schemes would perform over the proposed iROC Mars mission. The tool allows the user to apply constraints such as bandwidth allocations, spectral mask standard, maximum symbol rates, and optimum amplifier output power level. An analysis was conducted to select a set of modulation and coding schemes to maximize mission data return for the proposed mission. The spreadsheet-based tool includes all of the suggested modulation and coding combinations for the Mars mission (in Table 1 and Table 2) and calculates the signal to noise power spectral density ratio at the receiver on Earth given parameters for TWTA output power, antenna size and efficiency, path loss, Earth elevation angles, and average atmospheric loss conditions for each day of the proposed 2-year mission timeline. Given these parameters, the received signal-to-noise spectral density ratio is calculated as:

$$
\frac{P}{N_{0}}=E I R P_{s c}-L_{f s}-L_{a t m}+G / T(\theta)-L_{\text {pointing }}-k-M
$$

with terms defined as

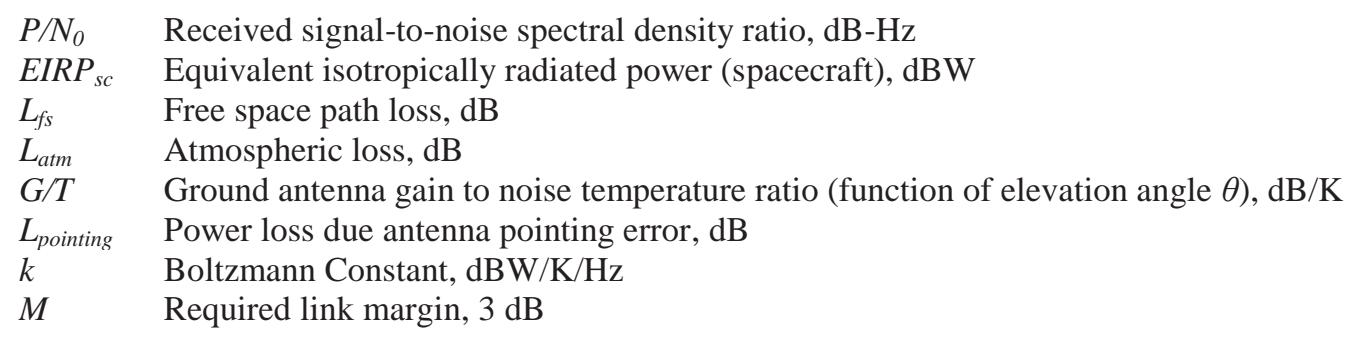

For a given mission analysis, the spacecraft EIRP, pointing loss, and required link margin are constant for the duration of the mission. Thus, only the free space path loss, atmospheric loss, and ground antenna G/T vary in the daily analysis that ensues. Contact time durations were calculated for each day of the mission at two different elevation angle ranges - between 10 and 30 degrees, and above 30 degrees, where atmospheric loss and G/T vary. Thus, the received signal-to-noise spectral density ratio is computed independently for each of the two elevation angle ranges for each day of the mission. For each elevation angle range, atmospheric loss and G/T are determined using data from the DSN telecommunications design handbook ${ }^{13,14}$ to ensure $90 \%$ availability of the link at the minimum elevation angle for that range, i.e. 10 and 30 degrees, respectively, using the yearly averages. This analysis is based on operation at a center frequency of $32.05 \mathrm{GHz}$, with an EIRP of $75.22 \mathrm{dBW}$, assuming $3 \mathrm{~dB}$ of excess link margin, $0.2 \mathrm{~dB}$ of pointing loss and use of the DSN 34-m DSS-25 antenna in Goldstone, CA.

In this link budget tool, concepts from science mission operations were also taken into account in order to obtain an accurate model for the mission data return. A ratio of 0.98 was used to represent the number of real science data bits per transmitted frame bits. This number is based on the protocol overhead from CCSDS TM Data Link framing headers. ${ }^{15}$ The tool also has the capability to limit contact time durations to reflect time-sharing of ground resources with other space missions. For the results presented in this paper, contact times are limited to a maximum of 8 hours per day. Given a value for $\mathrm{P} / \mathrm{N}_{0}$, the modulation and coding will then be selected to optimize the total mission data return, which is calculated from the data rate, bandwidth allocation, and daily contact times. 


\section{A. Modulation}

The modulation scheme chosen for the mission affects the spectral efficiency of the link and the modulation order. Given that bandwidth and symbol rate is being constrained, this is especially important to include in the analysis. The modulation schemes considered in this study are listed in Table 1 . The -25dB spectral efficiency values were taken from the CMLP report ${ }^{6}$ for GMSK and offset quadrature phase shift keying (OQPSK) with varying squareroot-raised-cosine (SRRC) pulse-shaping parameters, as per SFCG convention for deep-space. ${ }^{8}$ The tool is designed to consider one modulation choice at a time for the duration of the mission, which is selectable though its interface to adjust appropriate parameters characteristic of that modulation choice. In this way, it is very easy to compare the performance of different modulation schemes for the analysis. The tool also produces data that can be used to select the best modulation on a day-by-day basis throughout the mission, which is also considered.

Table 1. Modulation schemes analyzed.

\begin{tabular}{|c|c|c|}
\hline Modulation Families & Pulse Shaping & -25dB Spectral Efficiency \\
\hline (Pre-coded) GMSK $(\mathrm{h}=0.5)$ & Gaussian, BT $=0.3 ; 0.5 ; 0.25$ & $0.86 ; 0.77 ; 0.91$ \\
\hline Offset QPSK & SRRC, roll-off factor $=1.0 ; 0.5 ; 0.2$ & $1.04 ; 1.37 ; 1.69$ \\
\hline
\end{tabular}

\section{B. Coding}

The coding family chosen affects the range of code rates and $\mathrm{E}_{\mathrm{b}} / \mathrm{N}_{0}$ 's at which the link can operate. The coding schemes considered in this study are listed in Table 2. The turbo code family only includes rate $1 / 2$ and below, but has a lower $E_{b} / N_{0}$ requirement at these lower rates. The LDPC code family only includes rate $1 / 2$ and above with higher $\mathrm{E}_{\mathrm{b}} / \mathrm{N}_{0}$ requirements. $\mathrm{E}_{\mathrm{b}} / \mathrm{N}_{0}$ values used were taken from data published in the CMLP report for a bit-error-rate requirement of $10^{-8}$.

Table 2. Coding schemes analyzed.

\begin{tabular}{|c|c|c|c|}
\hline Code Family & Code Rate & Block Size (bits) & Fb/NO Requirement (dB) \\
\hline \multirow{4}{*}{ CCSDS Turbo } & $1 / 6$ & $8 \mathrm{k}$ & -0.02 \\
\cline { 2 - 4 } & $1 / 4$ & $8 \mathrm{k}$ & 0.27 \\
\cline { 2 - 4 } & $1 / 3$ & $8 \mathrm{k}$ & 0.58 \\
\cline { 2 - 4 } & $1 / 2$ & $8 \mathrm{k}$ & 1.3 \\
\hline \multirow{3}{*}{ CCSDS LDPC } & $1 / 2$ & $16 \mathrm{k}$ & 1.03 \\
\cline { 2 - 4 } & $2 / 3$ & $16 \mathrm{k}$ & 1.85 \\
\cline { 2 - 4 } & $4 / 5$ & $16 \mathrm{k}$ & 2.82 \\
\hline
\end{tabular}

\section{Data Rate Determination}

In order to calculate achievable data rates for a given code, the excess link margin (above the $3 \mathrm{~dB}$ required margin) is equated directly to a data rate. Excess link margin is calculated by subtracting the $E_{b} / N_{0}$ requirement for a particular coding scheme from calculated $\mathrm{P} / \mathrm{N}_{0}$ for that day. In addition, data rates are also limited by available bandwidth. The maximum data rate for a given bandwidth allocation is calculated by multiplying that bandwidth allocation by the spectral efficiency of the chosen modulation and coding. The effective data rate is the lesser of the achievable data rate by link margin and the maximum data rate in the bandwidth allocation. This calculation is performed for every code variant considered in the study, and the highest resulting data rate is selected for use on that particular day, and with a corresponding error correction code. The maximum bandwidth considered in the analysis is $500 \mathrm{MHz}$, the entire width of the Ka-Band deep space research return allocation ${ }^{16}$.

Figure 3 shows normalized total data return versus bandwidth limit for LDPC and Turbo codes, taking into account the entire 2-year mission. It can be seen that Turbo codes perform better than LDPC for this total mission data return when the bandwidth limit is greater than $80 \mathrm{MHz}$. For example, at a bandwidth limit of $200 \mathrm{MHz}$, the addition of Turbo coding capability to the LDPC results in a 15\% increase in total mission data return.

In addition, Figure 3 shows the diminishing returns associated with an increased bandwidth allocation. For example, a mission operating with a bandwidth allocation of $165 \mathrm{MHz}$ will return about $90 \%$ of the data that a mission with a $500 \mathrm{MHz}$ allocation would use. It is important to realize that while this bandwidth limit increases, the 
actual bandwidth used throughout the mission will vary. During the power limited phase of the mission, the larger bandwidth allocation is not in use, and thus the large allocation is not required during these periods. However, for deep space missions, spacecraft frequency allocations are generally fixed; therefore an assignment is retained and occupied for the duration of the mission.

Given a $200 \mathrm{MHz}$ allocation, the percentage of bandwidth used over the course of the mission for the LDPC only and Turbo-or-LDPC scenarios is shown in Figure 4. The option to use Turbo codes in addition to LDPC codes provides greater data return, but with greater utilization of the bandwidth assigned to the mission. The average daily bandwidth utilization if both LDPC and Turbo coding are allowed is more than twice that of using LDPC alone. Similarly, the period of time where usage exceeds $50 \%$ of the allocation is much longer when use of Turbo coding is an option. In this case, the period spans approximately 1 year of the mission. When LDPC is the only choice, the period spans approximately 4 months.

As previously stated, missions generally are assigned a fixed frequency and bandwidth allocation. So, while the use of Turbo coding necessitates increased bandwidth usage within the allocation, this usage is not a penalty within NASA's current concept of operations.

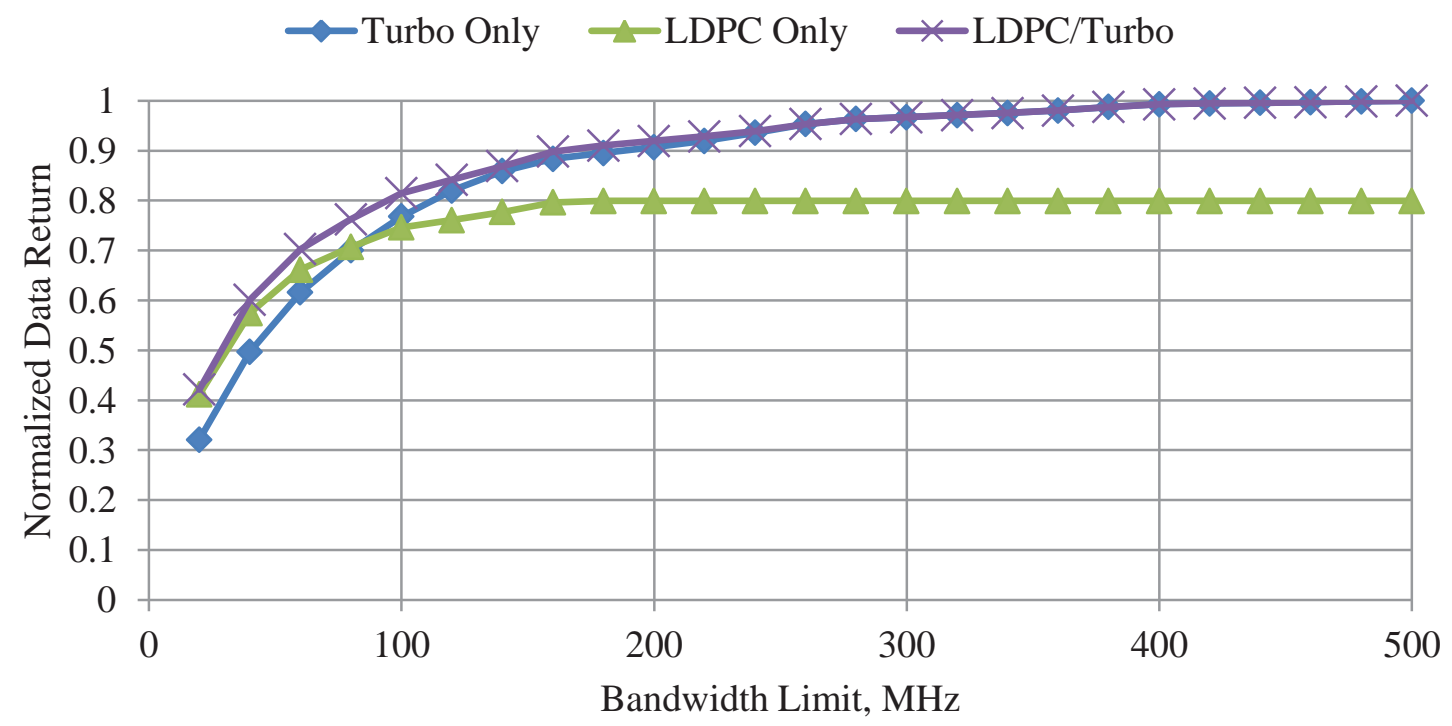

Figure 3. Comparison of total 2-year mission data return for various coding options.

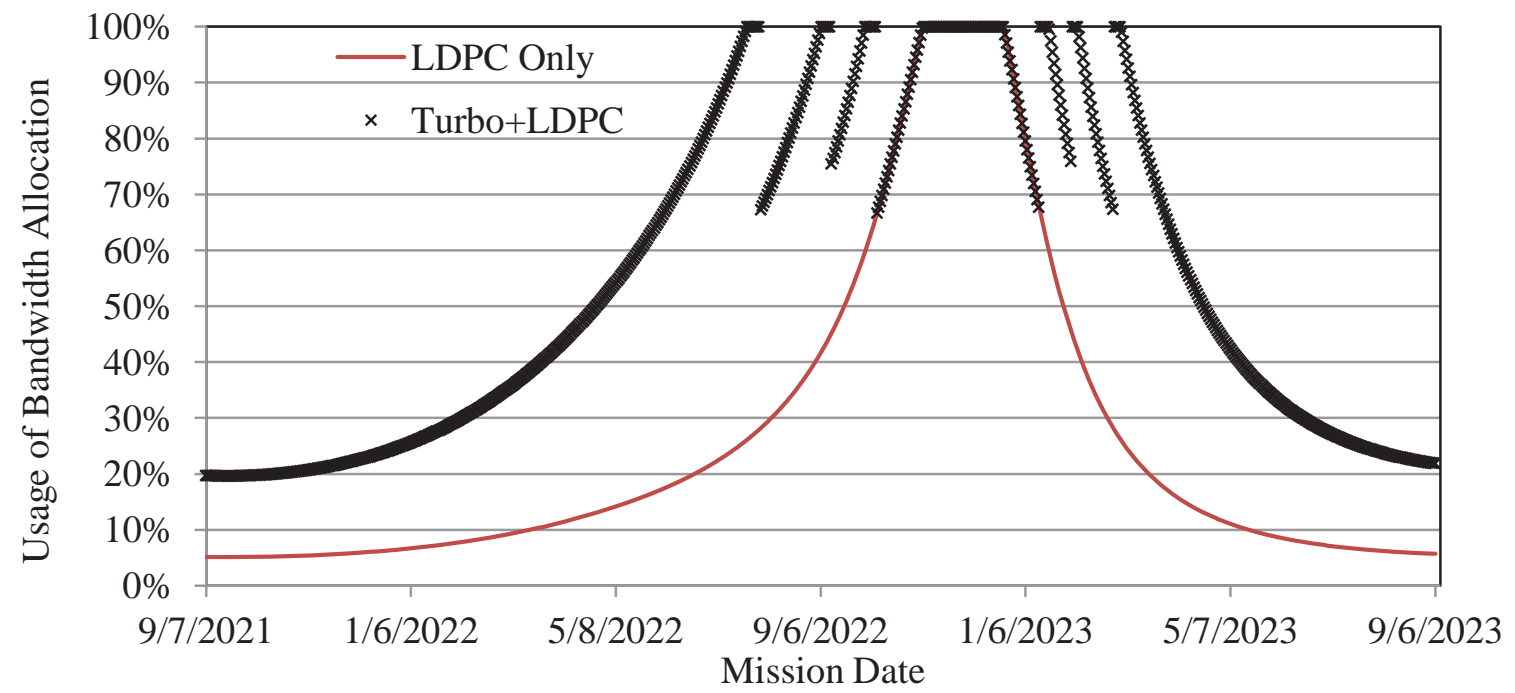

Figure 4. Percentage of Spectrum Allocation used throughout mission (with $200 \mathrm{MHz}$ limit) 


\section{Data Return Profile}

The chart in Figure 5 shows four mission data return profiles for a link using pre-coded GMSK (BT=0.25) modulation and bandwidth limits of $60 \mathrm{MHz}, 100 \mathrm{MHz}, 200 \mathrm{MHz}$, and $300 \mathrm{MHz}$. For these scenarios, the coding is selected to provide the highest data rate for a given contact time and $\mathrm{G} / \mathrm{T}$ range, permitting use of any variant of code rates and block sizes included in Table 2. In this plot, the daily data return trace has been smoothed using a 7day moving average. As seen in this mission profile, the effect of the bandwidth limitation is to constrain the maximum achievable data rate for the portion of the mission where Mars and Earth are closer. It is also possible for data rates to be limited by technology capabilities. For example, the maximum sample rate of the fastest space-rated DAC applies a bandwidth-like constraint to the link by limiting the maximum symbol rate. These other considerations are important when selecting a waveform and are discussed in further detail in section E.

Choppiness in these profiles is a result of switching code rates as the link enters and exits the bandwidthconstrained regimes throughout the mission. As such, the code rates used in each situation depend on the allocated bandwidth. The data return profiles presented in Figure 5 all use rate-1/6 Turbo code during the power constrained parts of the mission, which occur over several months at the beginning and the end. With a $250 \mathrm{MHz}$ allocation, the code rate never exceeds rate- $1 / 3$ over the course of the mission. With any lesser allocation, however, LDPC rate-1/2 is utilized. With allocations below $140 \mathrm{MHz}$, the links also use rate-2/3 LDPC, and below $85 \mathrm{MHz}$, also rate-4/5 LDPC.

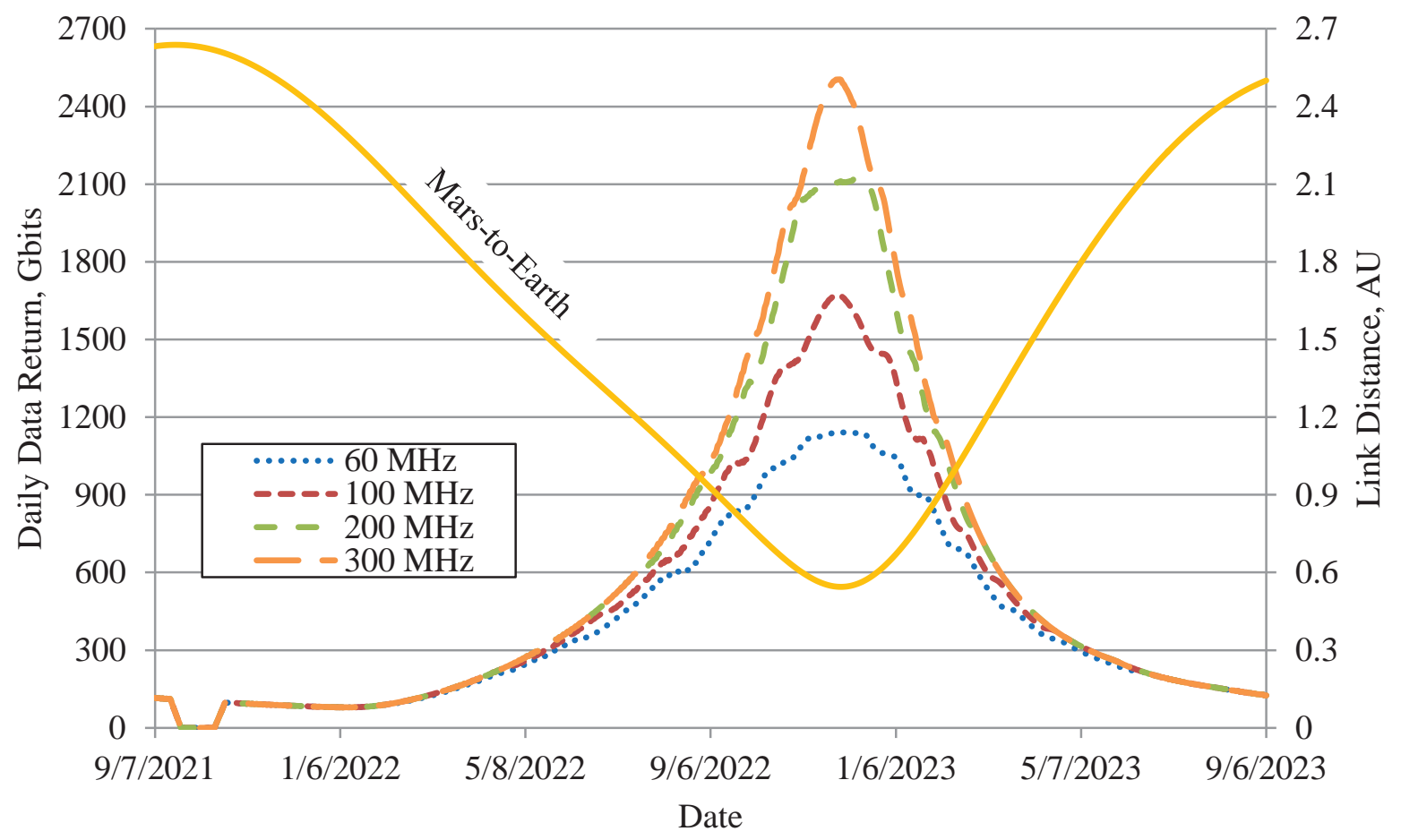

Figure 5. Mission data return profiles for various bandwidth allocations

\section{E. Software Defined Radio Technology}

An SDR architecture has been chosen to implement the selected waveform's modulation and coding for iROC. An SDR can include a reprogrammable field programmable gate array (FPGA) for increased flexibility in deep space missions, or the same design could also be implemented in a non-reprogrammable FPGA if power consumption is a consideration. An FPGA implementation is a convenient, lower cost alternative to an application specific integrated circuit (ASIC) assuming the FPGA can process signals fast enough for the desired application.

The data rate limiting technologies in an SDR include the FPGA and the DAC clock rates. Space-rated high speed DACs are capable of sampling rates up to $2.4 \mathrm{GHz}^{17}$. Analysis is presented using a DAC rate of $1800 \mathrm{MHz}$, based on the highest data interface speed supported by the space-qualified Xilinx Virtex-5QV. At this sampling rate, the maximum symbol rate is $450 \mathrm{MBd}$ when a minimum $4 \mathrm{x}$ oversampling factor is assumed for pulse shaping (see 
Table 3). This architecture assumes an in-phase/quadrature (I/Q) mixer located outside the FPGA for either OQPSK or GMSK modulation as shown in Figure 6. This example design with space qualified hardware demonstrates that symbol rates can go much faster than necessary for the iROC SDR, assuming a bandwidth constraint below 300 MHz. Though it has little effect for this mission scenario, the limiting nature of this constraint has been incorporated into the link analysis tool.

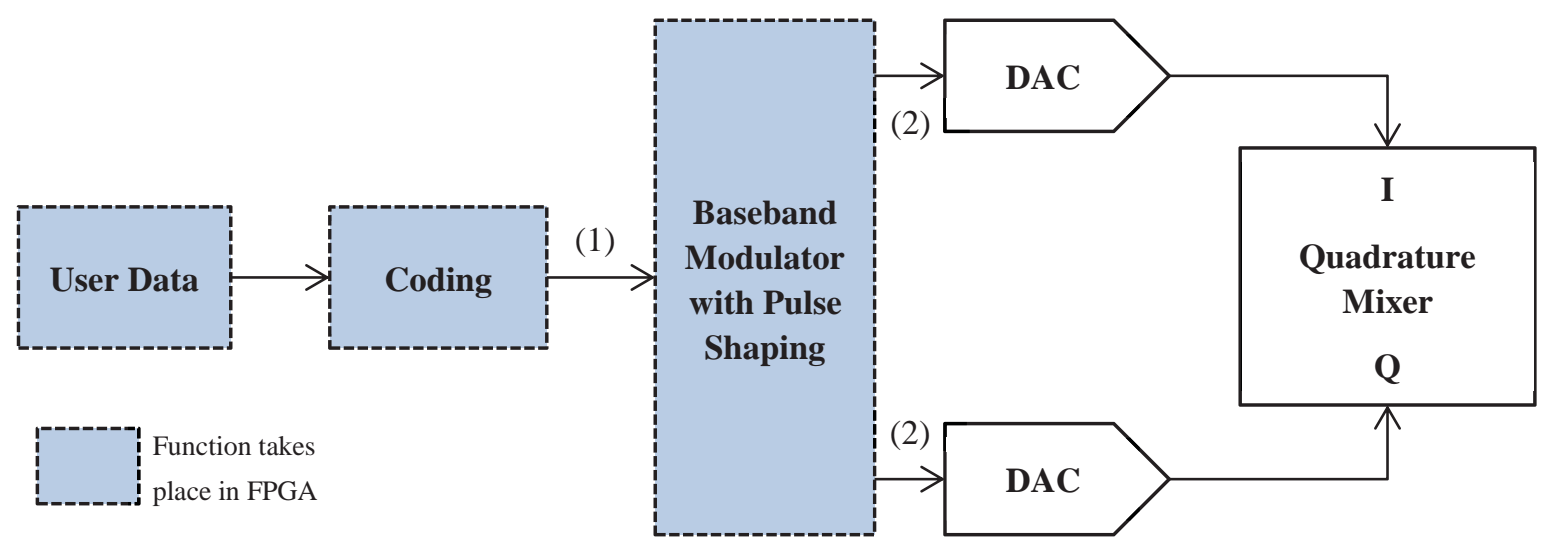

Figure 6. GMSK/OQPSK Transmitter Waveform Architecture

Table 3. SDR Clock Rate Calculations

\begin{tabular}{|c|c|c|}
\hline Modulation Type & GMSK & OQPSK \\
\hline Encoded Bit Rate [Mbps] (1) in figure & 450 & 900 \\
\hline Modulation Order [m-ary symbols] & 2 & 4 \\
\hline Bits/Symbol & 1 & 2 \\
\hline Symbol Rate [MBd] & 450 & 450 \\
\hline Pulse-shaping Samples/Symbol & 4 & 4 \\
\hline Sample Rate to DAC interface [MHz] (2) in the figure & 1800 & 1800 \\
\hline
\end{tabular}

\section{F. Ground Network Receiver Technology}

The DSN currently supports telemetry data rates with Turbo code rates $1 / 2,1 / 3$, and $1 / 4$ up to $1.6 \mathrm{Mbps}$, and up to 1.0 Mbps with a Turbo code rate $1 / 6^{18}$. The supported modulation includes BPSK, QPSK, and Offset QPSK. DSN upgrades to support LDPC codes and bandwidth efficient modulation (GMSK) would be necessary to achieve higher data returns, as well as faster processing hardware to support higher instantaneous data rates.

The Reconfigurable Wideband Ground Receiver ${ }^{19}$ has been developed and tested in a laboratory environment as well as in the field with the DSN ${ }^{20}$. It was tested with the X-Band MRO downlink at $1.5 \mathrm{MBd}$ and Lunar Reconnaissance Orbiter's Ka-Band downlink at 114 MBd. It supports demodulation of uncoded BPSK, QPSK, OQPSK, and GMSK (sub-optimally) at rates up to $320 \mathrm{MBd}$. This receiver demonstrates that rates enabled by the iROC system can be realistically supported up to a $320 \mathrm{MBd}$ rate with expected technology upgrades. However, further developments are needed to support high rate FEC decoding, which is an important consideration for iROC.

Market research for CCSDS-compliant Turbo decoders ${ }^{21}$ indicates that performance is currently about 10 Mbps. However, faster decoders have been produced for similar turbo codes in terrestrial communications standards. For example, one FPGA vendor has provided a similar binary turbo decoder IP core for the Third Generation Partnership Project-Long Term Evolution (3GPP-LTE) standard for licensing. Using supplied documentation ${ }^{22}$, data rates for this type of binary parallel concatenated turbo code is estimated at $183 \mathrm{Mbps}$. As the CCSDS turbo code has twice as many constituent encoder states, data rates near 90 Mbps are expected to be achievable for CCSDScompliant decoders if modernized to that of commercial state-of-the-art IP cores. Opportunities may also exist to instantiate multiple cores in a design. Unlike the CCSDS Turbo codes, implementations of CCSDS LDPC codes have already been created that approach gigabit speeds, thus decoding for use LDPC is not a concern for ground hardware. $^{23,24}$ 


\section{G. RF Amplifier Nonlinearity}

In the power constrained deep space environment, it is almost always advisable to operate the amplifier near peak power efficiency, which necessitates operating near saturation, and thus in a nonlinear region of the power curve. In the case of a continuous phase modulation such as GMSK, the amplifier can be operated at this peak power to no detriment. When using non-constant envelope modulations (i.e. with nonzero peak-to-average-power ratio (PAPR)), the TWTA output power is reduced in what is called output power back-off (OBO) in order to reduce distortion and spectral regrowth. The downside is that operating with an OBO comes at the expense of reduced $\mathrm{P} / \mathrm{N}_{0}$ in the communications link, and therefore reduced data rate. For this analysis, operation with OBO equal to the PAPR is considered for non-constant envelope modulations where distortions can be effectively removed by use of a predistortion filter. Alternatively, the system could deliberately operate with distortion in order to increase $\mathrm{P} / \mathrm{N}_{0}$. In fact, a recent study ${ }^{25}$ has shown that there is an optimum point of operation for the communications link at reduced OBO without using a pre-distortion filter. Based on an independent analysis using the noted method, and also taking into account reduced spectral efficiency when operating at the higher power levels, two other back-off points for pulseshaped OQPSK are considered in the analysis.

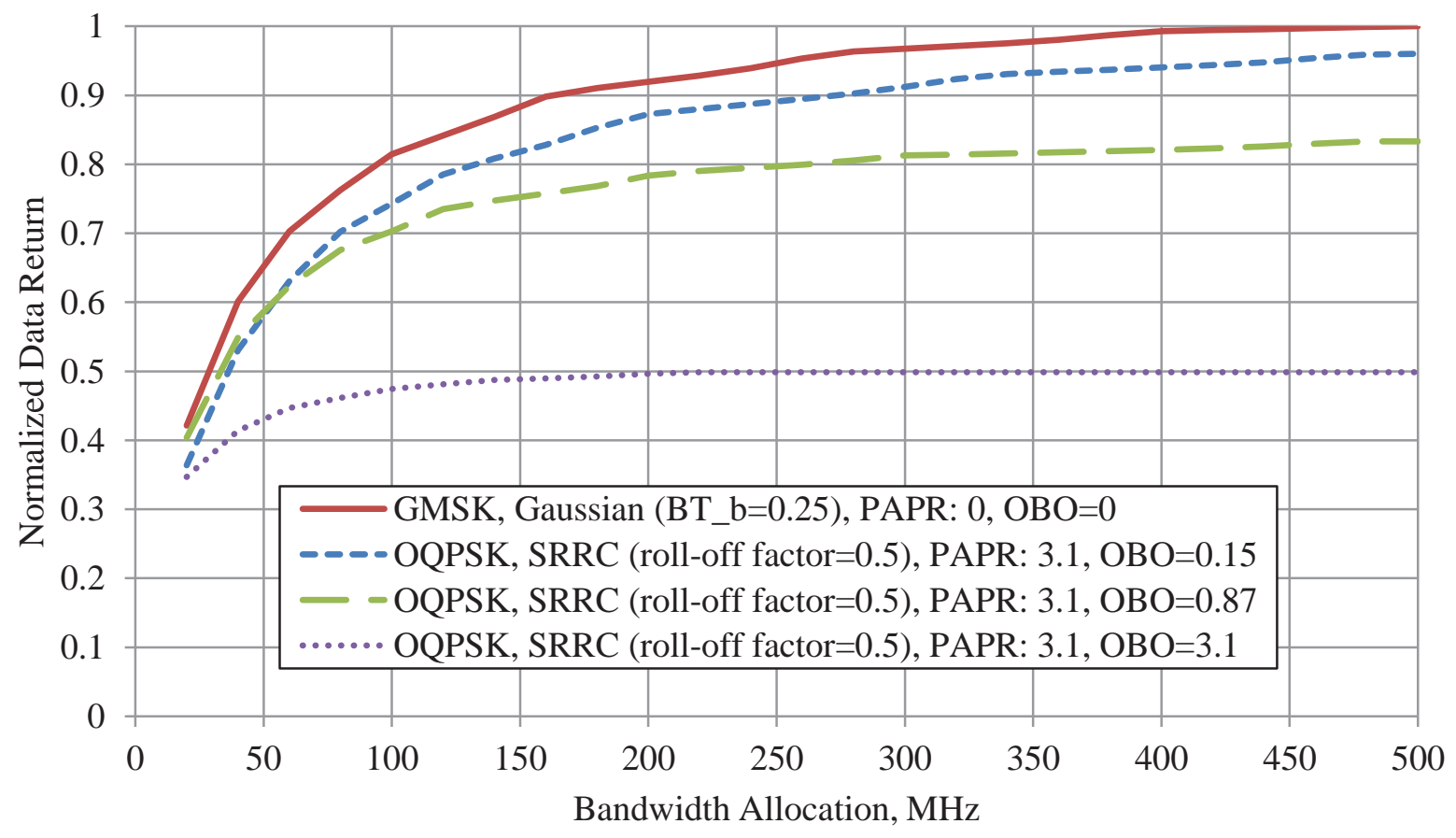

Figure 7. Effect of TWTA non-linearity on modulation performance with bandwidth limits

Figure 7 illustrates the effects of TWTA non-linearity on the data return of the OQPSK and GMSK modulations. The performance of the OQPSK modulation is shown at the three different OBO levels, and GMSK is shown without any OBO, as denoted in the legend. For the operating points where TWTA output power is fully backed off for filtered OQPSK, data return is significantly reduced, especially at higher bandwidth limits. In a more likely case of OQPSK operation when the OBO is less than one decibel or nearly zero, the performance is also slightly diminished compared to GMSK. Thus, usage of a constant-envelope modulation in this mission scenario demonstrates an advantage with respect to data return performance due to considerations of TWTA non-linearity.

\section{RF Modulation and Coding Recommendations}

Based on this study, the waveform recommended for use on iROC consists of a modulation of CCSDScompliant pre-coded GMSK paired with either a CCSDS rate-1/2 LDPC (16k block size) code, or any of the CCSDS-compliant Turbo codes (8k block size). For pre-coded GMSK, pulse shaping with a BT of 0.25 is advised. This differs from the GMSK with BT of 0.5 that is recommended in the CMLP report, because it is expected and advised that an optimal trellis receiver be implemented to remove inter-symbol interference associated with the more aggressive pulse shaping. In this case, the spectral efficiency and therefore data return provided by using a BT of 
0.25 will improve compared to that with $\mathrm{BT}$ of 0.5 . If the trellis receiver cannot be implemented at the required symbol rate, continued use of GMSK with the higher BT constant of 0.5 is advised.

Though LDPC coding is more spectrally efficient than turbo coding, inclusion of turbo encoders on the spacecraft transmitter is of negligible burden, and increases data return capability during the power-constrained portion of the mission. Therefore, the inclusion of CCSDS turbo codes is also recommended. If a bandwidth allocation below $140 \mathrm{MHz}$ were assigned, higher-rate LDPC codes would then be required to maximize mission data. Based on Figure 3, one might wonder why use of LDPC would be advised at all at higher bandwidth allocations. The primary reason is the small yet measurable additional coding gain and error floor performance of LDPC coupled with its high data rate decoding already supported in the ground receiver.

Based on the recommended waveform, the mission data return profiles are presented for certain example bandwidth allocations in Figure 5. Note that at the $100 \mathrm{MHz}$ bandwidth allocation, the inclusion of rate-2/3 LDPC coding is included in the recommendation. Table 4 below lists some other possible bandwidth allocations and corresponding data returns and peak data rates. The peak symbol rate of $273 \mathrm{MBd}$ at a bandwidth allocation of 300 $\mathrm{MHz}$ is within rates that are achievable with current SDR and ground receiver technology. Data return figures for allocations exceeding $300 \mathrm{MHz}$ are of greatly diminishing returns, and practically non-existent (less than 1\%) above a $400 \mathrm{MHz}$ allocation. Furthermore, missions are not realistically expected to be granted allocations above 300 $\mathrm{MHz}$, therefore data return estimates for these higher allocations is excluded from consideration in this recommendation.

Data return estimates using the day-by-day data rates for the recommended waveform are compared with the white Gaussian noise channel capacity information rates given by the classic limit derived by Shannon, again using $\mathrm{P} / \mathrm{N}_{0}$ values computed in the link tool. Contact times are again given by the mission model to the DSN Goldstone ground station. Across the entire range of bandwidth allocations, the data return difference remains approximately $130 \mathrm{~Tb}$. Performance as a percentage of capacity is also shown in Table 4. It increases diminishingly as bandwidth allocation increases. Finally, total data return estimates are compared to the demonstrated capability of MRO's Xband system, taken as $52 \mathrm{~Tb}$ for a 2-year mission based on previously stated yearly average data returns.

Table 4. Data Return Estimates

\begin{tabular}{|l|l|l|l|}
\hline Bandwidth Allocation (MHz) & $\mathbf{1 0 0}$ & $\mathbf{2 0 0}$ & $\mathbf{3 0 0}$ \\
\hline Data Return Estimate (Tb) & 269 & 303 & 319 \\
\hline Peak Data Rate (Mbps) & 60.5 & 76.2 & 84.5 \\
\hline Peak Symbol Rate (MBd) & 91.0 & 182 & 273 \\
\hline Selected Modulation & GMSK, BT=0.25 & GMSK, BT $=0.25$ & GMSK, BT=0.25 \\
\hline Selected Coding and Rates & Turbo: $1 / 6,1 / 4,1 / 3$ & Turbo: $1 / 6,1 / 4,1 / 3$ & Turbo: $1 / 6,1 / 4$, \\
& LDPC: $1 / 2,2 / 3$ & LDPC: $1 / 2$ & $1 / 3$ \\
\hline Shannon Capacity Data Return (Tb) & 399 & 434 & 448 \\
\hline Percentage of Shannon Capacity & $67.4 \%$ & $69.9 \%$ & $71.3 \%$ \\
\hline Compared to demonstrated MRO capability & $5.2 \mathrm{x}$ & $5.8 \mathrm{x}$ & $6.1 \mathrm{x}$ \\
\hline
\end{tabular}

\section{Conclusion}

In this study, the Ka-band link of an iROC SDR-based communications subsystem is analyzed for a proposed Mars orbiter mission. With the goal of maximizing total mission data return, the reliable RF link is designed to provide high data return capability in addition to the risk reduction it affords for infusion of promising optical communications capability into deep space missions. A tool was created to estimate total mission data return figures for a variety of possible waveform choices and practical constraints for future deep space missions by utilizing a day-by-day data return calculation. Using this tool, it was found that a ground-supported waveform consisting of GMSK modulation and turbo/LDPC codes that exist in current space communications standards performs optimally compared to other less spectrally-efficient and non-constant envelope waveforms, largely due to the reduction in link budget power from operating the TWTA in back-off mode for non-constant envelope waveforms.

In addition, by examining the bandwidth dependence of data return, it is apparent that any allocation above $300 \mathrm{MHz}$ provides greatly diminishing data returns for the proposed communications subsystem. Based on guidance from the SFCG, an allocation near $200 \mathrm{MHz}$ seems likely for the type of mission being considered. At that allocation level, projected Ka-band data return for the recommended waveform is $303 \mathrm{~Tb}$, a nearly $6 \mathrm{x}$ increase versus current data return capability from the X-band telecommunications subsystem used on MRO. The Ka-Band 
portion of iROC alone can provide significant data return capability to complement the data return projected for the optical link (not included in paper).

During the analysis, overlap between the Ka-Band and optical link waveforms was explored. The only sharing identified was for the separate RF and optical modulators and encoders to coexist on the same FPGA, for which integration is expected to provide savings in mass and power. Beyond the modulation and coding, expected areas for common hardware and processing include command and control, data handling, and usage of the Space Telecommunications Radio System (STRS) ${ }^{1}$ operating environment. As such, further development of an integrated RF/optical SDR architecture is required, including the addition of an RF uplink receiver.

Follow on work to this study could also include application of the tool to other missions, adding pass-by-pass capability in order to obtain even higher fidelity data return estimates, in which case further maximization of data return by optimizing operating points for availability per pass also becomes an option.

\section{References}

1 National Aeronautics and Space Administration. "Space Telecommunications Radio System (STRS) Architecture Standard.” NASA Technical Standard NASA-STD-4009. June 5, 2014.

2 D. Raible., "On the Physical Realizability of Hybrid RF and Optical Communications Platforms for Deep Space Applications." AIAA International Communications Satellite Systems Conference, AIAA, San Diego, CA, 2014.

3 J. Taylor, D. Lee, and S. Shambayati. "Mars Reconnaissance Orbiter Telecommunications," in DESCANSO Design and Performance Summary Series. Article 12. September 2006.

4 D. Agle, G. Webster., "Prolific NASA Mars Orbiter Passes Big Data Milestone," JPL Newsroom, Article 2013-324. URL: http://www.jpl.nasa.gov/news/news.php?release=2013-324 [cited 30 May 2014].

5 T. Bayer., "In-Flight Anomalies and Lessons Learned from the Mars Reconnaissance Orbiter Mission." IEEE Aerospace Conference, IEEE, Big Sky, MT, 2008, pp. 1-13

6 L. Deutch and F. Stocklin, "Coding, Modulation, and Link Protocol (CMPL) Study.” January 2008.

7 Space Frequency Coordination Group. "Recommendation SFCG 27-1R1: Efficient Spectrum Utilization for Space

Research Service, Deep Space (Category B), For Space-to-Earth Links in the 31.8-32.3 GHz Band.” June 15, 2011.

8 Space Frequency Coordination Group. "Recommendation SFCG 23-1: Efficient Spectrum Utilization For Space

Research Service, Deep Space (Category B), in the Space-To-Earth Link.” September 25, 2003.

9 The Consultative Committee for Space Data Systems. "TM Channel Coding Profiles.” Recommended Practice. 131.4M-1. July 2011.

${ }^{10}$ S. Shambayati, F. Davarian, D. Morabito., "Link design and planning for Mars Reconnaissance Orbiter (MRO) Kaband (32 GHz) telecom demonstration." IEEE Aerospace Conference, IEEE, Big Sky, MT 2005. pp.1559-1569

${ }^{11}$ S. Shambayati., "Ka-Band Telemetry Operations Concept: A Statistical Approach," Proceedings of the IEEE , vol.95, no.11, pp.2171,2179, Nov. 2007.

${ }^{12}$ M. Montagna. et. al."Maximisation of data return in Ka-band for interplanetary missions.” AIAA ICSSC/Ka- and Broadband Communications Conference.

${ }^{13}$ R. Sniffin, Ed., "DSN Telecommunications Link Design Handbook, 105, Rev. D, Atmospheric and Environmental Effects”, Jet Propulsion Laboratory, Pasadena, CA. September 15, 2009.

${ }^{14}$ R. Sniffin, Ed., "DSN Telecommunications Link Design Handbook, 104, Rev. G, 34-m BWG Stations

Telecommunications Interfaces”, Jet Propulsion Laboratory, Pasadena, CA. March 5, 2013.

15 The Consultative Committee for Space Data Systems. “TM Space Data Link Protocol.” Recommendation 132.0-B-1. July 2011

${ }^{16}$ R. Sniffin, Ed., "DSN Telecommunications Link Design Handbook, 201, Rev. B, Frequency and Channel Assignments”, Jet Propulsion Laboratory, Pasadena, CA, December 15, 2009.

${ }^{17}$ Texas Instruments, “14-Bit 2.4 Gsps Digital to Analog Converter.” Datasheet. December 2013.

${ }^{18}$ A. Bedrossian. "Deep Space Network Services Catalog." DSN No. 820-100, Rev. E. Jet Propulsion Laboratory, Pasadena, CA. December 2009.

${ }^{19}$ K. Andrews, A. Argueta, N. Lay, M. Lyubarev, E. Sigman, M. Srinivasan, and A. Tkacenko, "Reconfigurable Wideband Ground Receiver Hardware Description and Laboratory Performance." The Interplanetary Network Progress Report, vol. 42-180. February 2010.

${ }^{20}$ N. Lay, A. Argueta, A. Tkacenko, M. Srinivasan, and K. Andrews, "Reconfigurable Wideband Ground Receiver Field Testing.” The Interplanetary Network Progress Report, vol. 42-191. November 2012.

${ }^{21}$ TurboConcept. “TC6000 CCSDS Turbo Decoder Datasheet.” Revision 3. Datasheet. February 5, 2013.

22 Altera Corporation. “3GPP LTE Turbo Reference Design.” Application Note AN-505-2.1. January 2011.

${ }^{23}$ Zodiac Data Systems. "Cortex HDR XXL - High Data Rate Receiver.” Datasheet.

${ }^{24}$ RT Logic. "High Data Rate Receiver (T1200HDR).” Datasheet.

25 J.-P. Millerioux, C. Dudal. "CNES DVB-S2 simulations with non-linear amplification.” SLS-CS_13-17. Consultative Committee on Space Data Systems, Coding \& Synchronization Working Group. San Antonio, TX. October, 2013. 